\title{
ASTHMA
}

\section{Significant linkage to chromosome 12q24.32- q24.33 and identification of SFRS8 as a possible asthma susceptibility gene}

\author{
C Brasch-Andersen, Q Tan, A D Børglum, A Haagerup, T R Larsen, J Vestbo, T A Kruse
}

Thorax 2006;61:874-879. doi: 10.1136/thx.2005.055475

See end of article for authors' affiliations

Correspondence to Dr C Brasch-Andersen, Department of Biochemistry,

Pharmacology and

Genetics, Odense

University Hospital, DK-

5000 Odense C,

Denmark; charlotte.b. andersen@ouh.fyns-amt. $\mathrm{dk}$

Received7November 2005 Accepted 11 May 2006

Published Online First

31 May 2006

\begin{abstract}
Background: Asthma is a complex genetic disorder. Many studies have suggested that chromosome 12q harbours a susceptibility gene for asthma and atopy. Linkage on chromosome 12q24.21-q24.33 was investigated in 167 Danish families with asthma.

Methods: A two step procedure was used: (1) a genome-wide scan in one set of families followed by (2) fine scale mapping in an independent set of families in candidate regions with a maximum likelihood score $(M L S)$ of $\geqslant 1.5$ in the genome-wide scan. Polymorphisms in a candidate gene in the region on 12q24.33 were tested for association with asthma in a family based transmission disequilibrium test.

Results: An MLS of 3.27 was obtained at 12q24.33. The significance of this result was tested by simulation, resulting in a significant empirical genome-wide $p$ value of 0.018 . To our Knowledge, this is the first significant evidence for linkage on chromosome 12q, and suggests a candidate region distal to most previously reported regions. Three single nucleotide polymorphisms in splicing factor, arginine/ serine-rich 8 (SFRS8) had an association with asthma $(p \leqslant 0.0020-0.050)$ in a sample of 136 asthmatic sib pairs. SFRS8 regulates the splicing of CD45, a protein which, through alternative splice variants, has an essential role in activating T cells. T cells are involved in the pathogenesis of atopic diseases such as asthma, so SFRS8 is a very interesting candidate gene in the region.

Conclusions: Linkage and simulation studies show that the very distal part of chromosome 12q contains a gene that increases the susceptibility to asthma. SFRS8 could act as a weak predisposing gene for asthma in our sample.
\end{abstract}

B ronchial asthma [MIM 600807] is the most common chronic disease affecting children and young adults. The high prevalence in the population and assumed heterogeneity make it difficult to characterise the mode of inheritance and the genes involved. Furthermore, environmental factors contribute to the manifestation of asthma, ${ }^{1}$ and the disease is most likely caused by interactions between multiple genes and various environmental exposures.

Several association and linkage studies of asthma and atopy have suggested a number of candidate genes and chromosomal regions. To date, positional cloning has suggested six genes as possible candidates for asthma and atopy risk factors. ${ }^{2-7}$

Numerous studies have shown evidence for linkage to chromosome $12 \mathrm{q},{ }^{8-20}$ and the long arm of chromosome 12 is one of the most reproduced regions showing evidence for linkage to asthma. Even though none of these reports has reached genome-wide significance, accumulating evidence suggests that chromosome $12 \mathrm{q}$ may harbour one or more susceptibility genes for asthma and atopy.

There seems to be more than one region on $12 \mathrm{q}$ with evidence of linkage to asthma and atopy. Most groups have focused on regions around the interferon- $\gamma$ (IFNG) gene $12 \mathrm{q} 13-\mathrm{q} 15$ or distal to that region around $12 \mathrm{q} 21-\mathrm{q} 24{ }^{8-12}$ where a number of potential candidate genes for asthma and atopy map. These include the nitric oxide synthase gene (NOS1) and the signal transducer and activator of transcription 6 gene $(S T A T-6) .{ }^{21}$ Results from the various studies have been conflicting with regard to both significance and phenotypes.

Three studies have identified linkage to asthma even more distal on chromosome 12q (12q24.31-q24.33; table 1). These include a study by our group, an English group, and a
Japanese group. ${ }^{18-20}$ No genes have yet been investigated for an association with asthma and atopy in this region.

We chose a two step procedure using two Danish samples: (1) a genome-wide scan in one set of families followed by (2) fine scale mapping in a larger independent set of families in candidate regions with a maximum likelihood score (MLS) of $\geqslant 1.5$ in the genome-wide scan. The results of the genomewide scan have been published previously and suggest 12q24.21-q24.33 as a susceptibility locus for asthma. ${ }^{20}$

We also screened all coding single nucleotide polymorphisms (SNPs) in a functional gene (splicing factor, arginine/ serine-rich 8 (SFRS8) [MIM 601945]) from the region showing linkage in our study (confidence interval maximum MLS-1) and carried out association studies on the SNPs which were polymorphic, as well as testing five SNPs (intronic and upstream) for an association with asthma. SFRS8 regulates the splicing of CD45, also known as leucocyte common antigen [MIM 151460].22 ${ }^{23}$ CD45 exists in different isoforms depending on alternative splicing of exons 4, 5, and 6. The glycoprotein is an essential molecule for activating $\mathrm{T}$ cells ${ }^{24} 25$ by mediating cell-to-cell contacts and regulating protein tyrosine kinases involved in signal transduction. The activation of $\mathrm{T}$ cells is dependent on the different CD45 splice variants, ${ }^{24}{ }^{25}$ which are partly regulated by SFRS8. Mice and humans lacking CD45 expression are characterised by a block in T cell maturation. ${ }^{24} 25$ As T cells play a key role in the induction, regulation, and maintenance of the allergic inflammatory response, ${ }^{26}$ their activation could potentially be important in the development of atopic

Abbreviations: IBD, identity by descent; MLS, maximum likelihood score; SFRS8, splicing factor, arginine/serine-rich 8; SNP, single nucleotide polymorphism; TDT, transmission disequilibrium test 


\begin{tabular}{|c|c|c|c|}
\hline \multirow[b]{2}{*}{ Marker } & \multicolumn{2}{|l|}{ Position } & \multirow{2}{*}{ Cytogenetic } \\
\hline & $\overline{M b^{*}}$ & cMt & \\
\hline D12S2070 & 114.55 & 125.31 & $12 q 24.21$ \\
\hline $\mathrm{D} 12 \mathrm{~S} 2082$ & 116.30 & 130.94 & $\mathrm{q} 24.22$ \\
\hline D12S342 & 124.41 & 144.83 & $\mathrm{q} 24.31$ \\
\hline $\mathrm{D} 12 \mathrm{~S} 1659$ & 127.94 & 155.94 & $\mathrm{q} 24.32$ \\
\hline $\mathrm{D} 12 \mathrm{~S} 367$ & 128.49 & 159.59 & $\mathrm{q} 24.33$ \\
\hline $\mathrm{D} 12 \mathrm{~S} 1045 \S$ & 128.92 & 160.68 & $\mathrm{q} 24.33$ \\
\hline D12S97 & 128.92 & 160.68 & $\mathrm{q} 24.33$ \\
\hline D12S63 & 129.25 & 163.55 & $\mathrm{q} 24.33$ \\
\hline D12S392§ & 129.75 & 165.69 & $q 24.33$ \\
\hline SFRS8 & & & \\
\hline rs 1379049 & 130.86 ฯ & & $\mathrm{q} 24.33$ \\
\hline rs3782288 & 130.87 & & $\mathrm{q} 24.33$ \\
\hline rs930863 & 130.88 & & $\mathrm{q} 24.33$ \\
\hline rs 1051219 & 130.90 & & $\mathrm{q} 24.33$ \\
\hline rs1 107871 & 130.91 & & $\mathrm{q} 24.33$ \\
\hline rs 1051233 & 130.92 & & $\mathrm{q} 24.33$ \\
\hline rs 755437 & 130.93 & & $\mathrm{q} 24.33$ \\
\hline D12S357 & 131.77 & 168.79 & $\mathrm{q} 24.33$ \\
\hline \multicolumn{4}{|c|}{ 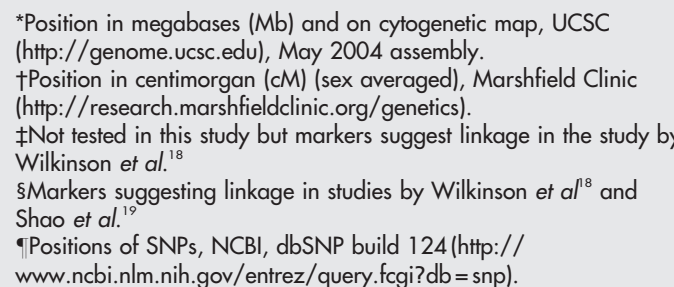 } \\
\hline
\end{tabular}

diseases such as asthma. SFRS8 is therefore a very interesting candidate gene for asthma susceptibility on chromosome $12 \mathrm{q} 24$.

\section{METHODS \\ Participants}

The combined sample consisted of 167 Danish families with asthma from two different asthma studies.

\section{Sample 1}

Sample 1 included 23 affected sib pair families and eight families with three affected children, a total of 39 independent sib pairs. For association studies, a further 13 families with one affected child were included. All probands were children suffering from allergic asthma. Allergic asthma was defined by clinical symptoms, the effect of standard asthma medication, and a positive specific immunoglobulin E (IgE) measurement to at least one of the 11 tested allergens (CAP RAST FEIA, Pharmacia). Clinical asthma was diagnosed according to standard criteria. ${ }^{27}$ The allergens tested were grass, birch, mugwort, olive, Pariaetaria, cat, dog, horse, mite (Dermatophagoides pteronyssinus and D farinae), and Cladosporium herbarum. Levels of $\geqslant 0.35 \mathrm{kUA} / \mathrm{l}$ ( $\geqslant$ class 1 ) were considered raised. The families have been described previously..$^{20}$

\section{Sample 2}

Sample 2 included 136 asthmatic sib pair families for linkage studies and a further 24 families with one asthmatic child were added for association studies. Seventy one sib pair families had sib pairs with a positive specific IgE test in addition to a diagnosis of asthma. The asthma inclusion criteria for sample 2 were clinically based on recurrent cough, wheezing and dyspnoea and a positive methacholine inhalation challenge identical to that used in a larger Danish study.$^{28}$ A positive response was equivalent to a provocative concentration causing a $20 \%$ fall in forced expiratory volume in 1 second $\left(\mathrm{FEV}_{1}\right)$ of $<8 \mathrm{mg} / \mathrm{ml}$. Subjects were considered to have a positive specific IgE test if specific IgE measurements were $\geqslant$ class 1 for at least one of the specific allergens tested (same as sample 1 except for olive and Pariataria) (CAP Phadiatop; Pharmacia \& Upjohn, Copenhagen, Denmark).

The study was approved by the Ethics Committee, Denmark, and informed written consent was obtained from all families before participation.

\section{Genotyping of microsatellite markers}

Genomic DNA was prepared from whole blood by standard methods. The following microsatellite markers were genotyped in both samples: D12S2070, D12S2082, D12S1659, D12S367, D12S1045, D12S63, D12S392 and D12S357 to fine map a region on chromosome 12q24 that was indicated in a genome-wide scan using sample 1 only. ${ }^{20}$ The mean distance between the markers in the candidate region (the last six markers) was $2.1 \mathrm{cM}$ (http://research.marshfieldclinic.org/ genetics/). Primer sequences were obtained from the Genome Database (www.gdb.org). The amplified products were separated on a $4 \%$ polyacrylamide gel using an ABI PRISM 377 automated DNA sequencer and analysed using ABI software (Applied Biosystems, Stockholm, Sweden).

\section{Genotyping of SNPs}

All SNPs were genotyped using the TaqMan method on ABI PRISM 7700. Primers and probes were designed using Primer Express software (Applied Biosystems); the sequences are shown in table 2. Primers were ordered from Invitrogen and probes from MWG and Applied Biosystems. Three SNPs (rs930863, rs1107871, rs755437) of SFRS8 were genotyped using Assays-on-Demand (Applied Biosystems) and primer/ probe sequences are therefore not available.

\section{Statistical analyses Linkage analysis}

Data from the affected sib pair families were analysed by non-parametric multipoint linkage analysis using the MLS approach. The computer program MAPMAKER/SIBS ${ }^{29}$ was used for calculation of multipoint maximum likelihood identity by descent (IBD), allowing for dominance variance. An increment step setting of five facilitated computing of MLS scores between each marker was used. If a family consisted of more than one affected sib pair, only independent pairs were used for calculations. Values for locus specific sibling relative risk ratio $\left(\lambda_{s}\right)$ for the susceptibility locus at peak MLS were calculated from the $\mathrm{z}_{0}$ parameter $\left(\lambda_{\mathrm{s}}=0.25\right.$ / $\left.\mathrm{z}_{0}\right)^{30}$

Simulation to determine the empirical significance of the MLS values obtained in this study was performed using Allegro 1.1. ${ }^{31}$ One thousand replicates of the entire autosomal genome were generated assuming no linkage using pedigrees from sample 1 and a marker distance of $5.8 \mathrm{cM}$ to mimic the original genome-wide scan. MAPMAKER/SIBS was used to analyse each of the replicates and regions with MLS values of $\geqslant 1.5$ were selected. Genotypes were then simulated in samples 1 and 2 at the selected regions but with a dense marker set (marker distance $2.9 \mathrm{cM}$ ); in sample 1 conditional on the original genotype distribution leading to the MLS $\geqslant 1.5$. MLS values were calculated for all the replicates and the number of replicates with MLS $\geqslant 3.27$ was counted to obtain a genome-wide empirical $\mathrm{p}$ value.

\section{Association analysis}

Transmission disequilibrium tests (TDTs) of polymorphisms and haplotypes in SFRSB and power estimations of the associations were performed using the FBAT and PBAT (Family Based Association Test) programs. ${ }^{32}{ }^{33}$ The TDT method makes statistical inferences by examining the conditional distribution of a score statistic, S, constructed to summarise the genotype and marker values in the affected 
Table 2 Primer and probe sequences for SNPs tested in SFRS8

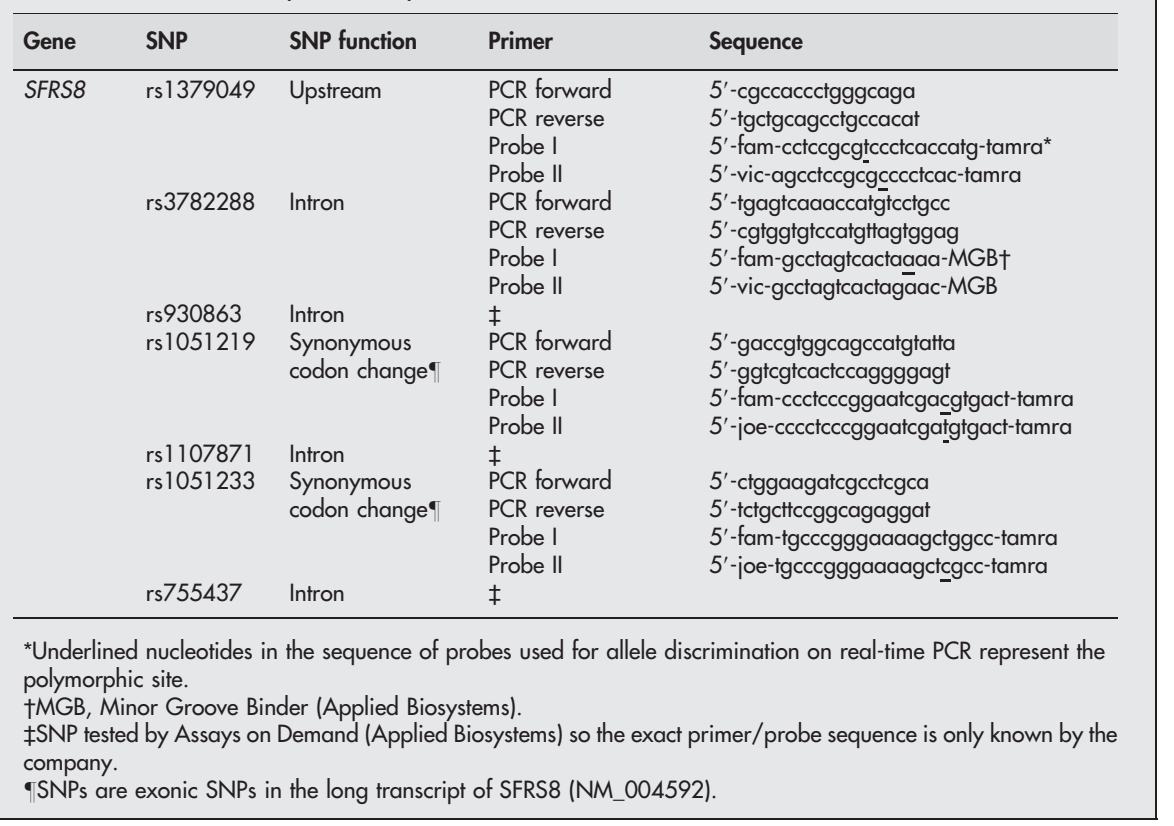

siblings. Most importantly, the method offers calculation of the empirical variance for the test statistics in the case of presence of linkage to account for sibling genotype correlation for families with more than one child, ${ }^{34}$ which is the case for most of our data.

\section{RESULTS}

\section{Linkage studies}

A genome-wide scan of sample 1 suggested chromosome 12q24.21-q24.33 to be a candidate region for asthma in the Danish population. ${ }^{20}$ We therefore genotyped both samples 1 and 2 (total of 167 pedigrees) in this region, using additional markers. Figure 1 shows the results from multipoint analyses of the two samples combined and for samples 1 and 2 individually using MAPMAKER/SIBS. Four markers had an MLS value of $>2.5$; the highest MLS value of 3.27 occurred between markers D12S63 and D12S392 when the two combined samples were analysed. Linkage analyses of sample 1 alone resulted in an MLS of 2.15 at marker D12S392 and

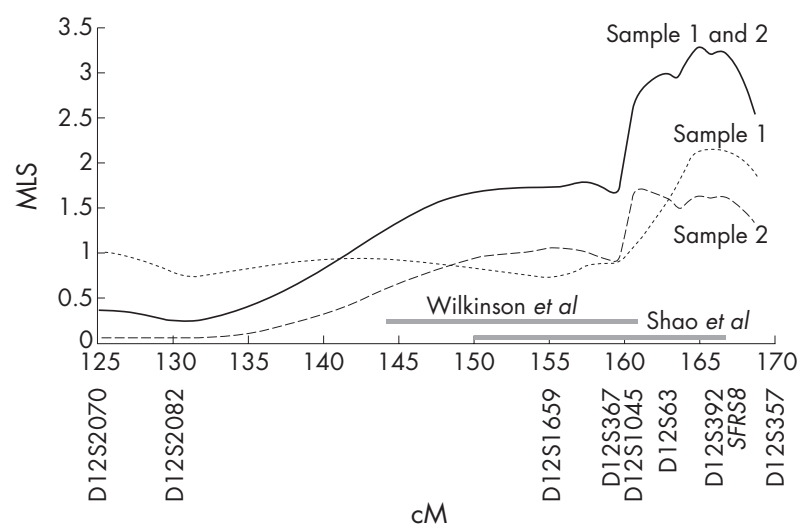

Figure 1 Linkage results from multipoint analyses for asthma on chromosome 12q. The grey bars illustrate the approximate location (due to limited availability of data) of linkage reported by Wilkinson et $a f^{18}$ and Shao et al. ${ }^{19}$ The position of the linkage signals from these two studies were adjusted for marker position according to the genetic map from Marshfield to be comparable to the linkage result in the Danish population. MLS, maximum likelihood score. the 136 affected sib pair families in sample 2 had an MLS of 1.71 at D12S1045 supported by nearby markers.

At the location of the maximum MLS, the IBD sharing between affected siblings was $62 \%$ for the combined sample, compared with the null hypothesis of $50 \%$. The information content in the point showing maximum MLS was 90\% (95\% information for the flanking markers). The locus specific sibling relative risk ratio $\left(\lambda_{\mathrm{s}}\right)$ for the susceptibility locus at peak MLS was 1.9 in the combined sample. The results of the simulation studies showed that the probability of obtaining an MLS value of $\geqslant 3.27$ by fine mapping the peak regions from the first genome scan was 0.018 , given the pedigree structure of both data sets and assuming no linkage. The IBD sharing $(61 \%)$ was maintained in the candidate region if samples 1 and 2 combined were subclassified into groups of sib pairs with a positive specific IgE test in addition to asthma (data not shown).

\section{Association studies}

Association analysis was performed on the microsatellite genotype data and resulted in a minimum $\mathrm{p}$ value of 0.009 (D12S1659). However, this result was not significant after correction for multiple testing of eight markers with an average of 10 alleles each.

Variations in SFRS8 - a positional and functional candidate gene-were chosen for the association studies. An additional 13 families with one affected child from sample 1 and 24 families with one asthmatic child from sample 2 were included in the association studies. Association with asthma was tested in a family based TDT design, using all sibs from the families, and showed that three SNPs (rs1051219, rs 1051233, rs755437) in SFRS8 (fig 2) were significantly associated with asthma (table 3 ). The strongest association

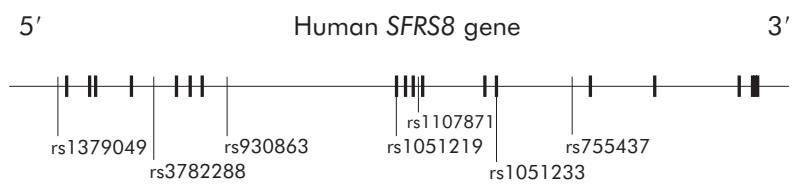

Figure 2 Gene structure of SFRS8 indicating the location of all SNPs included in the association study. 
Table 3 Results of transmission disequilibrium test (TDT)

\begin{tabular}{|c|c|c|c|c|c|c|c|c|c|c|c|c|c|c|c|c|}
\hline \multicolumn{2}{|l|}{ SFRS8* } & \multicolumn{5}{|c|}{ Sample 1} & \multicolumn{5}{|c|}{ Sample 2} & \multicolumn{5}{|c|}{ Sample 1 and 2} \\
\hline & Model & Allele & Fam $\ddagger$ & $\mathrm{S} \S$ & $E(S) \Phi$ & 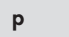 & Allele & Fam & $\mathbf{S}$ & $E(S)$ & $p$ & Allele & Fam & $\mathbf{S}$ & $E(S)$ & $\mathbf{p}$ \\
\hline \multirow[t]{3}{*}{ rs1379049 } & Additive & $\mathrm{g}$ & 19 & 20 & 18 & 0.50 & $\mathrm{~g}$ & 52 & 50 & 56 & 0.26 & $g$ & 71 & 70 & 74 & 0.51 \\
\hline & Recessive & g & 1 & & & $\star *$ & $g$ & 9 & & & $* *$ & g & 10 & 4 & 4.75 & 0.69 \\
\hline & Recessive & $a$ & 19 & 14 & 16.5 & 0.39 & $a$ & 52 & 49 & 43.25 & 0.23 & $a$ & 71 & 63 & 59.75 & 0.56 \\
\hline \multirow[t]{3}{*}{ rs3782288 } & Additive & $g$ & 19 & 48 & 50 & 0.50 & $g$ & 52 & 143 & 136 & 0.19 & $g$ & 71 & 191 & 186 & 0.42 \\
\hline & Recessive & $\mathrm{g}$ & 19 & 14 & 16.5 & 0.39 & $\mathrm{~g}$ & 52 & 50 & 43.75 & 0.19 & $g$ & 71 & 64 & 60.25 & 0.50 \\
\hline & Recessive & $a$ & 1 & & & ** & $a$ & 10 & 4 & 4.75 & 0.69 & $a$ & 11 & 4 & 5.25 & 0.53 \\
\hline \multirow[t]{3}{*}{ rs930863 } & Additive & $\mathrm{c}$ & 28 & 65 & 64.5 & 0.90 & c & 105 & 211 & 214.5 & 0.66 & c & 133 & 276 & 279 & 0.74 \\
\hline & Recessive & c & 23 & 19 & 19 & 1.00 & c & 80 & 63 & 58.75 & 0.46 & c & 103 & 82 & 77.75 & 0.52 \\
\hline & Recessive & $a$ & 11 & 7 & 7.5 & 0.81 & $a$ & 57 & 46 & 38.25 & 0.11 & $a$ & 68 & 53 & 45.75 & 0.17 \\
\hline \multirow[t]{3}{*}{ rs1051219 } & Additive & $t$ & 26 & 63 & 64 & 0.78 & $t$ & 85 & 184 & 190.5 & 0.35 & $t$ & 111 & 247 & 254 & 0.34 \\
\hline & Recessive & $t$ & 23 & 20 & 20.25 & 0.94 & $t$ & 79 & 58 & 58 & 1.00 & $t$ & 102 & 78 & 78.25 & 0.97 \\
\hline & Recessive & c & 6 & & & ** & c & 29 & 23 & 16.5 & $\begin{array}{l}0.050 \\
(0.12) \dagger \dagger\end{array}$ & c & 35 & 27 & 19.75 & $\begin{array}{l}0.043 \\
(0.10)\end{array}$ \\
\hline \multirow[t]{3}{*}{ rs1107871 } & Additive & $g$ & 28 & 56 & 61 & 0.25 & $g$ & 110 & 222 & 224 & 0.80 & $g$ & 138 & 278 & 285 & 0.45 \\
\hline & Recessive & $\mathrm{g}$ & 24 & 16 & 17.5 & 0.64 & $\mathrm{~g}$ & 86 & 62 & 62 & 1.00 & $g$ & 111 & 78 & 79.5 & 0.82 \\
\hline & Recessive & a & 15 & 12 & 8.5 & 0.14 & a & 58 & 40 & 38 & 0.68 & a & 73 & 52 & 46.5 & 0.30 \\
\hline \multirow{3}{*}{ rs1051233 } & Additive & c & 26 & 62 & 64.5 & 0.48 & c & 83 & 187 & 192.5 & 0.41 & c & 109 & 249 & 257 & 0.29 \\
\hline & Recessive & c & 23 & 19 & 20.5 & 0.64 & c & 78 & 61 & 60.25 & 0.89 & c & 101 & 80 & 80.75 & 0.91 \\
\hline & Recessive & $g$ & 5 & & & ** & $g$ & 22 & 18 & 11.75 & $\begin{array}{l}0.025 \\
(0.079)\end{array}$ & $g$ & 27 & 22 & 14.75 & $\begin{array}{l}0.018 \\
(0.057)\end{array}$ \\
\hline \multirow[t]{3}{*}{ rs755437 } & Additive & c & 29 & 54 & 56 & 0.63 & c & 112 & 239 & 217 & $\begin{array}{l}0.0068 \\
(0.017)\end{array}$ & c & 141 & 293 & 273 & $\begin{array}{l}0.028 \\
(0.050)\end{array}$ \\
\hline & Recessive & c & 21 & 12 & 14.75 & 0.34 & c & 82 & 76 & 58.25 & $\begin{array}{l}0.0020 \\
(0.0079)\end{array}$ & c & 103 & 88 & 73 & $\begin{array}{l}0.020 \\
(0.043)\end{array}$ \\
\hline & Recessive & $t$ & 16 & 11 & 11.75 & 0.77 & $t$ & 65 & 38 & 42.25 & 0.40 & $t$ & 81 & 49 & 54 & 0.38 \\
\hline \multicolumn{17}{|c|}{$\begin{array}{l}\text { *Ref Seq NM_004592. } \\
\text { †Only association to on } \\
\text { †Number of informative } \\
\text { SFBAT score statistics. } \\
\text { "Expected FBAT score st } \\
\text { alleles transmitted, giver } \\
{ }^{* *} \text { Association not tested }\end{array}$} \\
\hline
\end{tabular}

was found with the only intronic SNP of the three-rs755437 $(\mathrm{p} \leqslant 0.002)$-in a recessive model in sample 2 , which means that more affected offspring were homozygous for allele $\mathrm{C}$ than was expected by chance. The association with asthma for this SNP remained $(p \leqslant 0.0079)$ when calculated using the empirical variance (that is, correcting for linkage). Allele $C$ of SNP rs755437 also showed significant association in an additive model $(\mathrm{p} \leqslant 0.0068$, corrected $\mathrm{p} \leqslant 0.017)$. This association for allele $\mathrm{C}$ of rs755437 was also seen when the two samples were combined $(\mathrm{p} \leqslant 0.028)$. In the recessive model (for the combined sample) 88 homozygous probands for allele $\mathrm{C}$ were observed compared with 73 expected by chance $(\mathrm{p} \leqslant 0.02$, table 3$)$. A weak association with asthma was also seen for the SNPs rs 1051219 and rs 1051233, both of which are located in exons $(\mathrm{p} \leqslant 0.043$ and $\mathrm{p} \leqslant 0.018$, respectively) when testing the full sample.

The software PBAT estimates the conditional power of the results in the association study for every SNP. All significant associations in our study have a power of $\geqslant 0.98$ except the association of genotype CC for SNP rs1051219 in sample 2 only (PBAT power 0.05). The remaining SNPs tested in SFRS8 failed to show an association with asthma in our sample (table 3).

A p value of 0.0068 (corrected for linkage 0.013) was obtained when the three SNPs with significant associations were combined into haplotypes and tested for an association with asthma in sample 2 for the haplotype t-c-t. Significantly fewer $\mathrm{t}$-c-t haplotypes were transmitted to asthmatics than expected. The opposite haplotype (c-g-c) was also associated with asthma, despite the very few informative families (H5, table 4). A significant association for haplotypes 2 and 5 was also seen in the combined sample. Analysing the association of the haplotype in a phenotypic subgroup of sample 2 (patients with both asthma and increased specific IgE) resulted in a slightly more significant association, despite the fact that fewer families were included $(p \leqslant 0.0019$, corrected $\mathrm{p}$ value 0.0040, fewer haplotypes transmitted compared with the null hypothesis; data not shown).

In addition to the seven SNPs, four additional SNPs in SFRS8 were genotyped in our study-rs1051314, rs1982528, rs1051207 (all giving rise to a non-synonymous codon change) and rs3759110 (upstream from gene). None of the four additional SNPs was found to be polymorphic in our sample and this was later confirmed by the SNP databases for rs 1051314 and rs1051207.

\section{DISCUSSION}

In this study we investigated an asthma susceptibility locus at chromosome 12q24.32-q24.33 in the Danish population. Evidence for linkage in this region was obtained from a genome-wide scan of part of the samples ${ }^{20}$ and fine scale mapping was carried out with a denser set of markers in the full set of families including 136 additional sib pair families. A gene from the region was investigated for association by SNP genotyping using a total of 787 individuals including 379 asthmatics.

Multipoint linkage analysis using MAPMAKER/SIBS on the whole data set yielded an MLS score of 3.27 between markers D12S63 and D12S392, which shows that the distal region on $12 \mathrm{q}$ harbours a risk gene for the development of asthma.

Several groups have obtained suggestive evidence for linkage between $12 \mathrm{q}$ and asthma and atopy. ${ }^{8-19}$ Most of these groups have focused on the central part of the long arm on chromosome 12 and have not investigated the most distal part of the chromosome in detail. Only two other groups reported suggestive evidence for linkage to 12q24.33. Shao and co-workers suggested possible linkage to asthma using 19 families in the same region as in our study (table 1). ${ }^{19}$ Wilkinson et al also reported linkage at 12q24.33 at marker 
Table 4 Association between SFRS8 haplotypes and asthma

\begin{tabular}{|c|c|c|c|c|c|c|c|}
\hline \multirow[b]{2}{*}{ Haplotype } & \multirow[b]{2}{*}{ Frequencyt } & \multirow[b]{2}{*}{$\mathrm{N}$} & \multicolumn{3}{|l|}{ SNPs* } & \multirow[b]{2}{*}{ p value $\ddagger$} & \multirow[b]{2}{*}{ Power§ } \\
\hline & & & rs 1051219 & rs 1051233 & rs755437 & & \\
\hline \multicolumn{8}{|l|}{ Sample 2 } \\
\hline $\mathrm{Hl}$ & 0.46 & 102 & $t$ & c & c & $0.096(0.13)$ & $1(1)$ \\
\hline $\mathrm{H} 2$ & 0.26 & 88 & $t$ & c & $t$ & $0.0068(0.013)$ & $1(0.95)$ \\
\hline $\mathrm{H} 3$ & 0.19 & 77 & c & $\mathrm{g}$ & $t$ & $0.83(0.85)$ & $1(1)$ \\
\hline $\mathrm{H} 4$ & 0.042 & 24 & c & c & c & $0.64(0.67)$ & $0.14(0.20)$ \\
\hline H5 & 0.026 & 13 & c & $\mathrm{g}$ & c & $0.0047(0.043)$ & $1(0.62)$ \\
\hline \multicolumn{8}{|c|}{ Samples $1+2$} \\
\hline $\mathrm{HI}$ & 0.49 & 130 & $t$ & c & c & $0.20(0.23)$ & $1(1)$ \\
\hline $\mathrm{H} 2$ & 0.26 & 109 & t & c & $t$ & $0.016(0.026)$ & $1(1)$ \\
\hline $\mathrm{H} 3$ & 0.20 & 103 & c & $\mathrm{g}$ & $t$ & $0.69(0.72)$ & $0.79(0.65)$ \\
\hline $\mathrm{H} 4$ & 0.026 & 25 & c & c & c & $0.54(0.58)$ & $1(0.89)$ \\
\hline $\mathrm{H} 5$ & 0.017 & 14 & c & $g$ & c & $0.0049(0.044)$ & $0.05(0.20)$ \\
\hline
\end{tabular}

*Haplotype analysis only given for the three SNPs showing association with asthma individually.

†Haplotypes with frequency $<0.015$ are not shown.

$\mathrm{H}_{0}=$ no linkage and no association. Numbers in parentheses are $\mathrm{p}$ values corrected for linkage: $\mathrm{H}_{0}=$ linkage and no association. Significance and power are calculated using PBAT.

$\S$ Conditional power estimated by PBAT.

Results from sample 1 are not given as none of the SNPs showed association with asthma.

D12S97 using an asthma score. ${ }^{18}$ D12S97 is located in the same position as D12S1045 on the genetic map (table 1) used in our study and in the study by Shao et al, and had an MLS value of 2.7 in our study. This indicates that our results support the results of these two earlier studies. ${ }^{18}{ }^{19}$ Shao et al ${ }^{19}$ also found evidence to support linkage to the marker D12S392. There is therefore increasing evidence for an asthma susceptibility region on the very distal part of chromosome 12q. Linkage to the region 12q24.32-q24.33 was also tested in another smaller Danish sample in parallel to this study but no evidence for linkage was found. ${ }^{35}$

To evaluate the significance of our results we conducted a simulation study. Many studies only report point wise $p$ values at the location of the maximal lod score, which does not indicate the risk of type I errors on a genome-wide level. We simulated a full genome fine mapping study, in a very conservative way, using the sample and obtained a genomewide empirical p value of 0.064 for an MLS value of 3.27, which is close to the borderline $\mathrm{p}$ value of 0.05 . However, this is too conservative as full genome fine mapping of the whole sample was not conducted. Our simulation, which mimics the interdependent two step procedure, had a significance

\section{chr. 12}

$$
128922350
$$

129922350 130922350 131922350

D12S1045| D12S63| D12S392|

Marshfield markers

DI2S1045I D12S631 DI2S3921

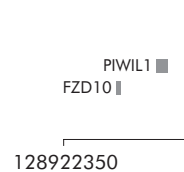

Reviewed
RAN I
EPIM ए

129922350

Figure 3 Reviewed Ref Seq genes located in the region showing evidence of linkage to asthma (maximum MLS-1). The coordinates are in $\mathrm{Mb}$ and extracted from UCSC, May 2004 assembly. FZD10, Frizzled 10 [MIM 606147]; PIWIL, piwi-like 1 [MIM 605571]; EPIM, epimorphin isoform 2 [MIM 132350]; RAN, member RAS oncogene family [MIM $601179] ;$ SFRS8, splicing factor arginine/serine-rich 8 isoform [MIM 601945]; MMP17, matrix metalloproteinase 17 preproprotein [MIM 602285]; PUS1, pseudouridylate synthase 1 isoform 2 [MIM 608109]; EP400, ElA binding protein p400 [MIM 606265] and GALNT9, polypeptide $\mathrm{N}$-acetylgalactosaminyltransferase 9 [MIM 606251]. Expanding the region to maximum MLS-1.5 does not include more reviewed Ref Seq genes. level of $p=0.018$ for an MLS value of $\geqslant 3.27$ by fine mapping peak regions from the first genome-wide scan.

In an attempt to investigate the effect of a narrower phenotype definition, we analysed the sib pairs from sample 2 who had a diagnosis of asthma and a positive specific IgE test. This reduced the total sib pair number to 112 but did not change the IBD sharing, indicating that the susceptibility locus is not specific for atopic asthma.

Several candidate genes for asthma susceptibility on chromosome 12q have been tested for association including IFNG, STAT6 and NOSI with conflicting results. ${ }^{21}$ The linkage results of the present study point to genes located distal to previously tested genes. SFRS8 maps within the region showing linkage in our study (confidence interval of maximum MLS-1) and was thus a potential candidate gene in the Danish sample. The gene codes for a splice factor which not only regulates its own splicing but also the splicing of CD45, ${ }^{22}{ }^{23}$ an important molecule in the activation process of T cells. ${ }^{24}{ }^{25}$ The activation of $\mathrm{T}$ cells depends on different splice variants of CD45. ${ }^{24} 25 \mathrm{~T}$ cells are involved in the pathogenesis of atopic diseases, ${ }^{26}$ but their exact role has still not been fully elucidated.

We tested 11 SNPs in SFRS8, seven of which were polymorphic in our samples and were tested in a family based TDT design. An association with asthma was found for three SNPs-rs1051219, rs1051233 and rs755437. The strongest association was seen for rs755437 where allele C was transmitted to affected offspring significantly more often than expected by chance in the additive model for both sample 2 and the combined samples $(p \leqslant 0.0068$ and 0.028 , respectively). Furthermore, homozygosity for allele $\mathrm{C}$ in rs755437 was observed in 88 cases compared with 73 expected by chance for the combined sample $(p \leqslant 0.020)$; the trend was even more pronounced for sample 2 $(p \leqslant 0.0020)$. These results were also statistically significant after correction for linkage, which means $\mathrm{H}_{0}=$ linkage and no association $^{34}(\mathrm{p} \leqslant 0.043$ and $\mathrm{p} \leqslant 0.0079$, respectively). All except one of the significant associations between SNPs in SFRS8 and asthma had a conditional power of $\geqslant 0.98$ and the positive association with asthma was also seen in our haplotype analysis of SFRS8.

Interestingly, increased expression by eosinophils of panCD45 and the splice variant CD45RO was observed in asthmatics than in controls. ${ }^{36}$

The association results for the individual SNPs and for the haplotypes indicate that SFRS 8 acts as a weak predisposing gene for asthma. 
When evaluating significant association studies, it is important to keep in mind that the literature reports numerous primary studies of significant associations but few associations have been reproduced. Additional studies are therefore necessary to determine whether SFRS8 is a weak susceptibility gene for asthma. However, we cannot rule out the possibility that the observed association could be due to linkage disequilibrium to a nearby gene.

Chromosome 12q24.32-q24.33 contains many other genes that could contribute to the susceptibility to asthma (fig 3), of which matrix metalloproteinase 17 (MMP17) would be a plausible candidate since another metalloproteinase (ADAM33) has been reported to underlie asthma. ${ }^{2}$ Two other genes (PLA2GIB and SCARBI) were tested for association with asthma in our population because of their position in the region that earlier showed evidence of linkage to an asthma score. ${ }^{18}$ PLA2GIB has also shown association with high total IgE levels in another study. ${ }^{37}$ The SNPs tested (rs5634, rs5888, rs5892) are located in exons but do not give rise to an amino acid change. We did not find evidence for association for these SNPs.

In summary, both the simulations and the replication of previous positive linkage results in a larger set of subjects suggest that we have identified an asthma susceptibility region for the Danish population distal on chromosome 12q. Association was furthermore found between asthma and SFRS8, a positional and functional candidate gene for atopy and asthma.

\section{ACKNOWLEDGEMENTS}

The authors thank Jette Bøhrk, Dorte Larsen, and Helle Binderup for skilful technical work and Margrethe Krog for graphical help.

\section{Authors' affiliations}

C Brasch-Andersen, Q Tan, T R Larsen, T A Kruse, Department of Biochemistry, Pharmacology and Genetics, Odense University Hospital, University of Southern Denmark, Odense, Denmark

A D Børglum, Institute of Human Genetics, University of Aarhus, Aarhus, Denmark

A Haagerup, Department of Paediatrics, County Hospital, Sygehus Viborg, Denmark

J Vestbo, Danish Epidemiology Science Centre, Institute of Preventive Medicine, Copenhagen University Hospital, Copenhagen \& Department of Cardiology and Respiratory Medicine, Hvidovre Hospital, Hvidovre, Denmark

This project was funded by the Danish Allergy Research Centre; Institute of Clinical Research, University of Southern Denmark; Hørslev Fonden; The Danish Lung Association; Direktør Jakob Madsen and Hustru Olga Madsen Fond; and the $\mathrm{E}$ and $\mathrm{H}$ Alstrup Fond.

Competing interests: CB-A, QT, TRL, ADB, AH and TAK declare no competing interests. JV has had no income related to asthma or genetics from any private organisation within the last 5 years and has no other conflicts of interests. He has, however, received both research funds and speaker's honoraria from GlaxoSmithKline, AstraZeneca, Pfizer and Boehringer-Ingelheim in relation to chronic obstructive pulmonary disease (COPD), which he does not consider a conflict of interest.

\section{REFERENCES}

1 Nolte H, Backer V, Porsbjerg C. Environmental factors as a cause for the increase in allergic disease. Ann Allergy Asthma Immunol 2001;87:7-1 1.

2 Van Eerdewegh P, Little RD, Dupuis J, et al. Association of the ADAM33 gene with asthma and bronchial hyperresponsiveness. Nature 2002;418:426-30.

3 Allen M, Heinzmann A, Noguchi E, et al. Positional cloning of a novel gene influencing asthma from chromosome 2q14. Nat Genet 2003;35:258-63.

4 Zhang Y, Leaves NI, Anderson GG, et al. Positional cloning of a quantitative trait locus on chromosome 13ql4 that influences immunoglobulin $E$ levels and asthma. Nat Genet 2003;34:181-6.

5 Laitinen T, Polvi A, Rydman P, et al. Characterization of a common susceptibility locus for asthma-related traits. Science 2004;304:300-4
6 Nicolae D, Cox NJ, Lester LA, et al. Fine mapping and positional candidate studies identify HLA-G as an asthma susceptibility gene on chromosome $6 \mathrm{p} 21$. Am J Hum Genet 2005;76:349-57.

7 Noguchi E, Yokouchi Y, Zhang J, et al. Positional identification of an asthma susceptibility gene on human chromosome 5q33. Am J Respir Crit Care Med 2005; 172: 183-8.

8 Barnes KC, Freidhoff LR, Nickel R, et al. Dense mapping of chromosome $12 q 13.12-q 23.3$ and linkage to asthma and atopy. J Allergy Clin Immunol 1999:104:485-91.

9 Blumenthal MN, Rich SS, King R, et al. Approaches and issues in defining asthma and associated phenotypes map to chromosome susceptibility areas in large Minnesota families. The Collaborative Study for the Genetics of Asthma (CSGA). Clin Exp Allergy 1998;28(Suppl 1):51-5.

10 Wist M, Fischer G, Immervoll T, et al. A genome-wide search for linkage to asthma. German Asthma Genetics Group. Genomics 1999:58:1-8.

11 Malerba G, Lauciello MC, Scherpbier T, et al. Linkage analysis of chromosome 12 markers in Italian families with atopic asthmatic children. Am J Respir Crit Care Med 2000;162:1587-90.

12 Yokouchi Y, Nukaga Y, Shibasaki M, et al. Significant evidence for linkage of mite-sensitive childhood asthma to chromosome 5q31-q33 near the interleukin $12 \mathrm{~B}$ locus by a genome-wide search in Japanese families. Genomics 2000;66:152-60.

13 CSGA. A genome-wide search for asthma susceptibility loci in ethnically diverse populations. The Collaborative Study on the Genetics of Asthma (CSGA). Nat Genet 1997; 15:389-92.

14 Ober C, Cox NJ, Abney M, et al. Genome-wide search for asthma susceptibility loci in a founder population. The Collaborative Study on the Genetics of Asthma. Hum Mol Genet 1998;7:1393-8.

15 Dizier MH, Besse-Schmittler C, Guilloud-Bataille M, et al. Genome screen for asthma and related phenotypes in the French EGEA study. Am J Respir Crit Care Med 2000;162:1812-8

16 Xu J, Postma DS, Howard TD, et al. Major genes regulating total serum immunoglobulin $\mathrm{E}$ levels in families with asthma. Am J Hum Genet 2000;67:1 163-73.

17 Raby BA, Silverman EK, Lazarus R, et al. Chromosome 12q harbors multiple genetic loci related to asthma and asthma-related phenotypes. Hum Mol Genet 2003:12:1973-9.

18 Wilkinson J, Grimley S, Collins A, et al. Linkage of asthma to markers on chromosome 12 in a sample of 240 families using quantitative phenotype scores. Genomics 1998;53:251-9.

19 Shao C, Suzuki Y, Kamada F, et al. Linkage and association of childhood asthma with the chromosome 12 genes. J Hum Genet 2004:49:115-22.

20 Haagerup A, Bierke T, Schiotz PO, et al. Asthma and atopy: a total genome scan for susceptibility genes. Allergy 2002;57:680-6.

21 Malerba G, Pignatti PF. A review of asthma genetics: gene expression studies and recent candidates. J App/ Genet 2005;46:93-104.

22 Sarkissian M, Winne A, Lafyatis R. The mammalian homolog of suppressorof-white-apricot regulates alternative mRNA splicing of CD45 exon 4 and fibronectin IIICS. J Biol Chem 1996;271:31106-14.

23 Lemaire R, Winne A, Sarkissian M, et al. SF2 and SRp55 regulation of CD45 exon 4 skipping during T cell activation. Eur J Immunol 1999:29:823-37.

24 Kishihara K, Penninger J, Wallace VA, et al. Normal B lymphocyte development but impaired T cell maturation in CD45-exon6 protein tyrosine phosphatase-deficient mice. Cell 1993;74:143-56.

25 Kung C, Pingel JT, Heikinheimo M, et al. Mutations in the tyrosine phosphatase CD45 gene in a child with severe combined immunodeficiency disease. Nat Med 2000;6:343-5.

26 Romagnani S. The role of lymphocytes in allergic disease. J Allergy Clin Immunol 2000; 105:399-408.

27 Nicklas RA. National and international guidelines for the diagnosis and treatment of asthma. Curr Opin Pulm Med 1997:3:51-5.

28 Vestbo J, Thomas W, the ASTHMAGEN study group. Asthma and genes in a Danish population: outline of an on-going study. Eur Respir Rev 2000;10:396-9.

29 Kruglyak L, Lander ES. Complete multipoint sib-pair analysis of qualitative and quantitative traits. Am J Hum Genet 1995:57:439-54.

30 Risch N. Linkage strategies for genetically complex traits. III. The effect of marker polymorphism on analysis of affected relative pairs. Am J Hum Genet 1990;46:242-53.

31 Gudbjartsson DF, Jonasson K, Frigge ML, et al. Allegro, a new computer program for multipoint linkage analysis. Nat Genet 2000;25:12-3.

32 Horvath S, Xu X, Laird NM. The family based association test method: strategies for studying general genotype-phenotype associations. Eur J Hum Genet 2001;9:301-6.

33 Lange C, DeMeo D, Silverman EK, et al. PBAT: tools for family-based association studies. Am J Hum Genet 2004;74:367-9.

34 Lake SL, Blacker D, Laird NM. Family-based tests of association in the presence of linkage. Am J Hum Genet 2000:67:1515-25.

35 Haagerup $A$, Borglum $A D$, Binderup $H G$, et al. Fine-scale mapping of type allergy candidate loci suggests central susceptibility genes on chromosomes $3 q, 4 q$ and Xp. Allergy 2004;59:88-94.

36 Blaylock MG, Lipworth BJ, Dempsey OJ, et al. Eosinophils from patients with asthma express higher levels of the pan-leucocyte receptor CD45 and the isoform CD45RO. Clin Exp Allergy 2003;33:936-41.

37 Barnes KC, Neely JD, Duffy DL, et al. Linkage of asthma and total serum lgE concentration to markers on chromosome 12q: evidence from Afro-Caribbean and Caucasian populations. Genomics 1996;37:41-50. 